Research Paper

\title{
Identification and Characterization of Wnt Signaling Pathway in Keloid Pathogenesis
}

\author{
Shinichi Igota1,2, Mamiko Tosa1,3, Masahiro Murakami³, Seiko Egawa1 , Hajime Shimizu¹, Hiko Hya- \\ kusoku4, Mohammad Ghazizadeh ${ }^{\circledR}$ \\ 1. Department of Molecular Pathology, Institute of Gerontology, Nippon Medical School, Kawasaki, Japan; \\ 2. Department of Plastic and Reconstructive Surgery, Higashi-totsuka Memorial Hospital, Yokohama, Japan; \\ 3. Department of Plastic and Reconstructive Surgery, Nippon Medical School, Musashi-kosugi Hospital, Kawasaki, Japan; \\ 4. Department of Plastic and Reconstructive Surgery, Nippon Medical School, Tokyo, Japan.
}

$\triangle$ Corresponding author: M. Ghazizadeh, MD, PhD. Department of Molecular Pathology, Institute of Gerontology, Graduate School of Medicine, Nippon Medical School, Kawasaki 211-8533, Japan. Tel: 81-44-733-1821 Ext. 8867 E-mail: ciem@nms.ac.jp.

(c) Ivyspring International Publisher. This is an open-access article distributed under the terms of the Creative Commons License (http://creativecommons.org/ licenses/by-nc-nd/3.0/). Reproduction is permitted for personal, noncommercial use, provided that the article is in whole, unmodified, and properly cited.

Received: 2012.10.08; Accepted: 2013.02.10; Published: 2013.02.15

\begin{abstract}
Keloid is characterized by fibroblastic cell proliferation and abundant collagen synthesis. Numerous studies have shown that the Wingless type (Wnt) signaling pathways play key roles in various cellular functions including proliferation, differentiation, survival, apoptosis and migration. The aim of this study was to clarify the role of Wnt signaling pathway in keloid pathogenesis. Primary fibroblast cultures and tissue samples from keloid and normal appearing dermis were used. The expression of Wnt family members, frizzled (FZD)4 receptor, receptor tyrosine kinase-like orphan receptor (ROR)2 and the Wnt signaling downstream targets, glycogen synthase kinase (GSK)3- $\beta$ and $\beta$-catenin were assessed using semi-quantitative RT-PCR, Western blot, or immunohistochemical methods. Of the Wnt family members, Wnt5a mRNA and protein levels were elevated in keloid fibroblasts (KF) as compared to normal fibroblasts (NF). A higher expression of $\beta$-catenin protein was also found in KF. No detectable levels of FZD4 receptor and ROR2 proteins were observed in both NF and KF. Functional analysis showed that treatment of $\mathrm{NF}$ and KF with recombinant Wnt5a peptide resulted in an increase in protein levels of total $\beta$-catenin and phosphorylated $\beta$-catenin at Ser33/37/Thr 4 I but no significant change in phosphorylated $\beta$-catenin at Ser45/Thr 4I positions. In addition, the expression of total GSK3- $\beta$ protein was not affected but its phosphorylated/inactivated form was increased in NF and KF. Our findings highlight a potential role for a $\mathrm{Wnt} / \beta$-catenin canonical signaling pathway triggered by Wnt5a in keloid pathogenesis thereby providing a new molecular target for therapeutic modulations.
\end{abstract}

Key words: Keloid pathogenesis, wound healing, Wnt signaling, Wnt5a, $\beta$-catenin, frizzled4, ROR2.

\section{Introduction}

Keloids are abnormal wound healing lesions of unknown etiology characterized by fibroblastic proliferation and excessive collagen deposition. Keloids do not usually regress spontaneously, and they tend to recur after excision. Many factors such as skin tension, wound infection, racial difference, and genetic predisposition have been implicated in the etiology of keloid lesions (1). Treatment of keloids currently includes surgery alone or in combination with corticosteroids or radiotherapy. In a randomized trial for keloid therapy, a combination of surgery and radiotherapy appeared to be more effective ( $12.5 \%$ relapse) 
than that of surgery and corticosteroid injections (33\% relapse) at 12 months after treatment (2). Recently, early postoperative electron beam irradiation has proved to be a well-tolerated and effective method in reducing the recurrence rate of keloid (3-6). However, unraveling the mechanism(s) behind the development of keloids is highly desirable in order to explore effective modes of therapy and prophylaxis for this lesion.

Many studies have shown that the Wingless type (Wnt) signaling pathway plays a key role in various cellular functions including proliferation, differentiation, apoptosis, survival, migration, and polarity, by regulating multiple intracellular signaling cascades $(7$, $8)$. These critical roles in development require tight regulation of this signaling pathway with over or under expression of target genes resulting in disease and cancer (9). Cell signaling via the Wnt/FZD pathway is highly conserved through evolution (10). The Wnt family is composed of 19 secreted glycoproteins (11) that act as ligands for the Frizzled (FZD) family of receptors (12-16). FZD receptors are members of the $G$ protein coupled receptor family and are found in multiprotein complexes $(17,18)$. In addition to FZD proteins, single-pass transmembrane proteins, such as low-density lipoprotein receptor-related protein 5 (LRP5), LRP6, receptor tyrosine kinase-like orphan receptor 1 (ROR1), ROR2, and atypical tyrosine kinase receptor (RYK), have been shown to act as Wnt receptors (19-22). The best characterized example of Wnt signaling is the Wnt/ $\beta$-catenin pathway. Traditionally, Wnt signaling is divided into canonical, i.e. $\beta$-catenin-dependent signaling and non-canonical or $\beta$-catenin-independent signaling. Non-canonical pathways are the cell polarity pathway or the $\mathrm{Ca} 2+$ pathway (7). Even though molecular details in the Wnt signaling pathway are still waiting for full understanding, basic concepts are known. Wnt binding to FZDs and co-receptors, e.g. the LRP family, namely LRP5/6, induces the activation of the disheveled (Dvl) signaling relay (23). Activation of Dvl in the $\beta$-catenin pathway results in inhibition of a destruction complex for $\beta$-catenin consisted of adenomatous polyposis coli (APC), axin, and glycogen synthase kinase 3 beta (GSK3- $\beta$ ). This in turn prevents proteasomal degradation of $\beta$-catenin, leading to cytoplasmatic stabilization of the protein and a final translocation to the nucleus where it exerts gene transcriptional modulation in cooperation with $\mathrm{T}$ cell factor (TCF)/lymphoid enhancer factor (LEF) family transcription factors (7, 11).

In the Wnt canonical pathway and when Wnt is not stimulated, $\beta$-catenin levels are kept at a minimum level through the destruction complex com- posed of APC, Axin, GSK3 $\beta$, and casein kinase 1 (CK1). In the nucleus, TCFs associate with transcriptional repressors to block target gene activation. When Wnt is stimulated, the destruction complex is destabilized, and $\beta$-catenin accumulates in the nucleus to activate transcription of TCF target genes. The accumulated $\beta$-catenin binds the transcription factor of the LEF/TCF family and converts them from repressors to activators, which triggers downstream gene transcription (24-26). The activity of $\beta$-catenin can also be regulated in the canonical Wnt pathway in a GSK3-independent manner as has been shown recently in Drosophila (27).

In the Wnt/non-canonical pathways, i) Calcium-induced calmodulin-dependent protein kinase II (CaMKII) TAK1-NLK pathway suppresses canonical Wnt signaling by inhibiting $\beta$-catenin-dependent transcription; ii) Planar cell polarity mediated by ROR2/CDC42 and also another pathway mediated by small GTPase (RHO and RAC), JNK, and CDC42 regulate gene expression.

There is increasing evidence to show that the canonical Wnt/ $\beta$-catenin signaling is involved in keloid pathogenesis (28). Sato (29) reported that TGF- $\beta$ induced the upregulation of Wnt/ $\beta$-catenin signaling in hypertrophic scar and keloid fibroblasts. Gene profiling of normal scar fibroblasts and KFs (30) as well as immunohistochemical examination of active areas of keloid tissues (31) revealed the decreased expression of secreted frizzled-related protein 1 (SFRP1), a known Wnt inhibitor. In addition, initial microarray profiling of human skin NF and KF performed in our laboratory also revealed significant down-regulation of SFRP1 in KF (unpublished data). However, the exact role of Wnt signaling in keloid pathogenesis still has not been clarified.

Activation of Wnt receptors can result in the activation of canonical "Wnt/ $\beta$-catenin," or "non-canonical" Wnt/Ca(2+) or Wnt/planar cell polarity" pathways. The aim of our study was to determine which Wnt signaling pathway is involved in keloid pathogenesis in order to facilitate development of novel treatment modalities.

\section{Materials and Methods}

\section{Tissue specimens}

Tissue samples from keloid $(\mathrm{n}=18)$ and residuals of normal skin tissues after reconstructive surgery $(n=10)$ were used. Informed consents had been obtained from all patients and the study was conducted according to the guidelines of the Institutional Review Board of the Nippon Medical School. The patients ranged in age from 20 to 67 years. The keloids had 
been developed after complete healing of wound injury or resolution of acne. No patient was on any form of medicine. Hematoxylin and eosin-stained sections from the samples were histologically examined to confirm characteristics of keloid or normal skin tissues.

\section{Antibodies and recombinant peptide}

Polyclonal rabbit antibodies were against Wnt5a (Lifespan Biosciences, Seattle, WA), phospho-beta-catenin Ser33/37/Thr41 and phospho-beta-catenin Ser45/Thr41 positions, and phospho-GSK3-beta Ser9 position (Cell Signalling Technology Inc., Beverly, MA), and monoclonal mouse antibodies were against FZD4 (R\&D Systems, Minneapolis, MN), ROR2 (Santa Cruz Biotechnology, Inc., LA), beta-catenin (Upstate Biotechnology, Waltham, MA), beta-actin (Sigma-Aldrich, St. Louis, MO). Monoclonal rabbit antibody was against human GSK3-beta (Epitomics, Burlingame, CA). The recombinant Wnt5a peptide was purchased from R\&D Systems Inc. (Minneapolis, MN).

\section{Cell culture and characterization}

Primary cultures of keloid fibroblasts (KFs, $n=3$ ) and normal dermal fibroblasts (NFs, $n=3$ ) were used. The location of keloid lesions was on the chest area. The KFs and NFs had been characterized in terms of the cell growth and collagen synthesis properties as reported previously (32). Early passages (4-7) of cells were cultured in Dulbecco's modified Eagle's medium (DMEM), supplemented with $10 \%$ fetal calf serum, penicillin $(100 \mathrm{IU} / \mathrm{ml})$, streptomycin $(100 \mu \mathrm{g} / \mathrm{ml})$, and amphotericin B $(0.25 \mu \mathrm{g} / \mathrm{ml})$ at $37 \mathrm{C}$ in a humidified 5\% CO2 incubator. The cultures were maintained until reaching 70 to $80 \%$ confluence. The cells were harvested by trypsinization $(0.25 \%$ trypsin in EDTA).

\section{RNA extraction}

Culture flasks $\left(75 \mathrm{~cm}^{2}\right)$ containing nearly $80 \%$ confluent KFs and NFs were prepared and the cells were harvested and subjected to RNA isolation using the RNeasy Midi Kit (Qiagen, Hilden, Germany) based on the manufacturer's instructions. Total RNA was treated with DNase and precipitated using 95\% ethanol before cDNA synthesis. Isolated RNA was electrophoresed through 1.0\% agarose-formaldehyde gels to verify the quality of the RNA, and RNA concentrations were determined by UV spectrophotometry.

\section{Semiquantitative RT-PCR}

From each sample, $1.5 \mu \mathrm{g}$ of total RNA was re- verse-transcribed using the gene-specific primers. The number of PCR cycles was optimized in each case to ensure that product intensity fell within the linear phase of amplification. The primer pair sequences were: Wnt1 forward 5'-CTA CTT CGA GAA ATC GCC CAA C-3' (nucleotides 1068-1090), reverse 5'-ACA GAC ACT CGT GCA GTA CGC-3' (nucleotides 1309-1330); product size 242-bp, Wnt2 forward 5'-GGG AAT CTG CCT TTG TTT ATG CCA-3' (nucleotides 617-641), reverse 5'-GAA CCG CTT TAC AGC CTT CCT GCC-3' (nucleotides 903-927); product size 287-bp, Wnt3 forward 5'-CAG CAG TAC ACA TCT CTG GGC TCA-3' (nucleotides 216-240), reverse 5'-CTG TCA TCT ATG GTG GTG CAG TTC-3' (nucleotides 409-433); product size 194-bp, Wnt4 forward 5'-CTG AAG GAG AAG TTT GAT GGT GCC-3' (nucleotides 804-828), reverse 5'-GTG GAA TTT GCA GCT GCA GCG TTC-3' (nucleotides 1097-1121); product size 294-bp, Wnt5a forward 5'-CTT CGC CCA GGT TGT AAT TGA AGC-3' (nucleotides 423-447), reverse 5'-CTC CCA AAA ACA GAG GTG TTA TCC-3' (nucleotides 695-719); product size 273-bp, Wnt6 forward 5'-CTT GGT TAT GGA CCC TAC CAG CAT C-3' (nucleotides 311-336), reverse 5'-CAC TGC AGC AGC TCG CCC ATA GAA-3' (nucleotides 610-634), product size 300-bp; Wnt7a forward 5'-AAT GCC CGG ACT CTC ATG AAC TG-3' (nucleotides 828-851), reverse 5'-ACG GCC TCG TTG TAC TTG TCC TTG A-3' (nucleotides 1012-1034); product size 185-bp; $\beta$-catenin forward 5'-ATG GAA GGT CTC CTT GGG ACTC-3' (nucleotides 1460-1481), reverse 5'-CTC CAC AAA TTG CTG CTG TGT C-3' (nucleotides 1954-1975); product size 495-bp. Glyceraldehyde 3-phosphate dehydrogenase (GAPDH; product size 450-bp) was used as the internal control. The RT-PCR was performed for $26 \mathrm{cy}-$ cles. The PCR bands were quantitated by densitometry using Quantity One Software (Bio-Rad Labs., Hercules, CA) and expressed relative to GAPDH. The results were recorded as mean \pm SEM of triplicate experiments.

\section{Western blot analysis}

Proteins were extracted from the subconfluent primary cultures of the three cases each of KFs and NFs. Pairs of cultured cells were subjected to the treatment with recombinant Wnt5a peptide at $3 \mu \mathrm{g} / \mathrm{ml}$ for 72 hours or left non-treated as controls. This dose was chosen based on our own preliminary studies on $\mathrm{NF}$ and KF cultures and also previous reports validating the maximal effectiveness of the same recombinant Wnt5a peptide at $3 \mu \mathrm{g} / \mathrm{ml}$ on human fibroblast proliferation (33). Equal amounts of protein $(20 \mu \mathrm{g} /$ lane $)$ were size fractionated on 
SDS-polyacrylamide gels, blotted onto an Immobilon polyvinylidene difluoride membranes (Bio-Rad Laboratories, Hercules, CA) in transfer buffer containing $192 \mathrm{mM}$ glycine, $25 \mathrm{mM}$ Tris- $\mathrm{HCl}, \mathrm{pH} \mathrm{8.3,20 \%} \mathrm{v/v}$ methanol, and $0.02 \%$ SDS), and incubated with blocking buffer ( $5 \%$ nonfat dry milk, $50 \mathrm{mM}$ Tris-HCl, $\mathrm{pH} 7.5,150 \mathrm{mM} \mathrm{NaCl}, 0.1 \%$ Tween-20) overnight at $4^{\circ} \mathrm{C}$. Duplicate series of membranes were prepared and incubated overnight at room temperature with 1:800 dilutions of primary antibodies against each total or phosphorylated form of proteins. After washes with Tris buffered saline with $0.1 \%$ Tween (50 mM Tris-HCl, pH 7.5, $150 \mathrm{mM} \mathrm{NaCl}, 0.1 \%$ Tween-20) buffer and treatment with corresponding alkaline phosphatase-conjugated secondary antibodies, the reactions were developed with the ProtoBlor NBT and BICP Color Development System (Promega, Madison, WI). Western blot bands were quantitated by densitometry measurements using the "Image J" software.

\section{Immunohistochemistry}

Immunohistochemical staining for Wnt5a, $\beta$-catenin, and GSK3- $\beta$ was performed on frozen or deparaffinized paraffin sections from the tissues using a method described previously (32). The primary antibodies were applied at 1:50 or 1:100 dilution overnight after appropriate antigen retrieval methods recommended by the manufacturers. Nonspecific binding sites in tissue sections were blocked with $10 \%$ normal serum of the corresponding secondary antibody's animal. For immunofluorescence staining, the sections were incubated with Alexa Fluor 594 (red) labeled donkey anti-rabbit secondary antibodies (Invitrogen, Inc., Carlsbad, CA) at 1:800 dilution for $2 \mathrm{~h}$ at room temperature, washed in phosphate-buffered saline (PBS) and mounted with or without 4',6-diamidino-2-phenylindole (DAPI) nuclear staining. For immunoperoxidase staining, the endogenous peroxidase was blocked with $0.3 \%$ hydrogen peroxide in distilled water for 30 minutes. After washes in PBS, nonspecific binding sites in tissues were blocked with $10 \%$ normal serum and the sections were incubated overnight with the primary antibody. After washes in PBS, appropriate biotinylated secondary antibodies were applied followed by washes in PBS and detection using a streptavidin-biotin-peroxidase staining kit (Histofine, Nichirei, Japan). Diaminobenzidine was used as chromogen. Nuclear counterstaining was with hematoxylin. Negative controls included omission of the primary antibody and its substitution with nonimmune sera. Fibroblastic cells with distinct brown immunostaining in the cytoplasm and/or nucleus were considered as positive. The staining results were semiquantitatively assessed by two observers using a method described previously with slight modification (34). In each case, five randomly selected microscopic fields at $200 \times$ magnification were screened and the number of positive and negative fibroblastic cells were recorded. At least 500 fibroblastic cells in each case were counted. The mean percentage of immunostained positive fibroblastic cells for normal and keloid fibroblasts was then calculated. The mean intra- and inter-observer variation was less than $7 \%$. The results were further classified as negative/weak, moderate, or strong, if $0-<25,25-50$, or $>50 \%$, respectively, of the fibroblastic cells showed distinct cytoplasm or nuclei staining.

\section{Statistical analysis}

The results were recorded as the mean \pm standard deviation (SD). Continuous data between two groups were compared using unpaired Student's $t$ test. The significance of immunohistochemical expression of Wnt5a and $\beta$-catenin was assessed using Fisher's exact test. A p-value of less than 0.05 was considered as significant.

\section{Results}

\section{Expression of Wnt family members in keloid and normal fibroblasts}

Measurement of mRNA level by a semiquantitative RT-PCR in the 3 representative cases each of NF and KF which were also utilized in the functional analysis in this study showed that from the Wnt family members 1 to 7 , Wnt5a was markedly $(p<0.05)$ and Wnt2 was sporadically expressed in KF as compared to NF (Fig. 1).

\section{Expression of Wnt5a in keloid and normal tissues}

To validate the result of Wnt5a mRNA level, we performed immunofluorescence and immunohistochemical stainings for Wnt5a in randomly selected 10 normal skin tissues and 18 keloid tissues. Both cytoplasmic and nuclear staining was observed (Fig. 2 A-D). Of the 10 normal skin tissues, 7 (70\%) showed negative/weak staining for Wnt5a (Table 1). In contrast, of the 18 keloid tissues, 14 (78\%) showed moderate to strong staining for Wnt5a. Overall, fibroblasts in keloid tissues showed a significantly higher expression of Wnt5a than those in normal tissues $(p=$ $0.013)$. As $\beta$-catenin is a well-known major player in the Wnt signaling pathways, we stained the same tissue sections for $\beta$-catenin as well and found a significantly higher expression of $\beta$-catenin in fibroblasts 
in $13(72 \%)$ keloid cases as compared to negative/weak staining in 7 (70\%) of normal tissues (Fig. $3 \mathrm{~A}$ and $\mathrm{B}$, Table $1 ; p=0.038$ ). We also verified the immunolocalization of FRZ4 and ROR2 receptors which are involved in non-canonical Wnt/ $\beta$-catenin signaling pathways and found no detectable reactions in both NF and KF (images not shown). Further semi-quantitative evaluation by Western blot analysis showed a markedly higher expression of Wnt5a and $\beta$-catenin and lower levels of FRZ4 and ROR2 in KFs versus NFs (Fig. 3C).

\section{Effect of Wnt5a recombinant peptide on $\beta$-catenin and GSK3- $\beta$}

To examine whether Wnt5a had an effect on $\beta$-catenin and GSK3- $\beta$, we treated NFs and KFs with recombinant Wnt5a peptide for 72 hours and measured total $\beta$-catenin, phosphorylated $\beta$-catenin at Ser45/Thr41 and at Ser33/37/Thr 41 positions, total GSK3- $\beta$ and phosphorylated GSK3- $\beta$ at Ser 9 position in both NFs and KFs. Both NFs and KFs exhibited a significant increase in the total $\beta$-catenin level (Fig. $4 \mathrm{~A})$, no significant change in phosphorylated $\beta$-catenin at Ser45/Thr41 (Fig. 4B) and a significant increase in phosphorylated $\beta$-catenin at Ser33/37/Thr 41 position (Fig. 4C). Also, there was no significant change in total GSK3- $\beta$ level (Fig. 5A) but phosphorylated/inactivated GSK3- $\beta$ level in both NFs and KFs was significantly increased (Fig. 5B).

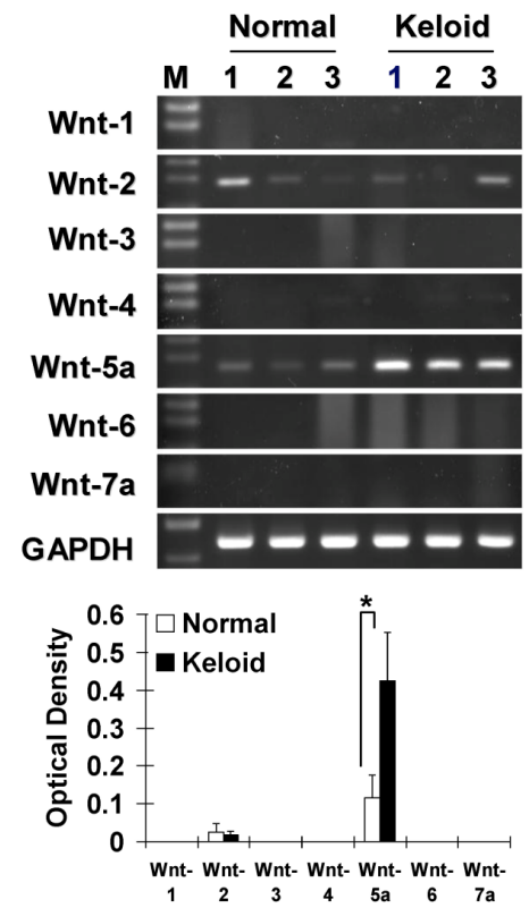

Fig I. Measurement of mRNA by RT-PCR in normal dermal fibroblasts (NFs) and keloid derived fibroblasts (KFs) with semiquantitative analysis shows the expression patterns of the Wnt family members. Note that Wnt5a mRNA was markedly expressed $(p<0.05)$ and $W n t 2$ was sporadically expressed in keloid fibroblasts compared to normal fibroblasts. The RT-PCR cycles were kept constant for all the reactions ( 26 cycles). GAPDH was used as an internal positive control. Data represent the mean \pm SD of three cases in each group of NF and KF.
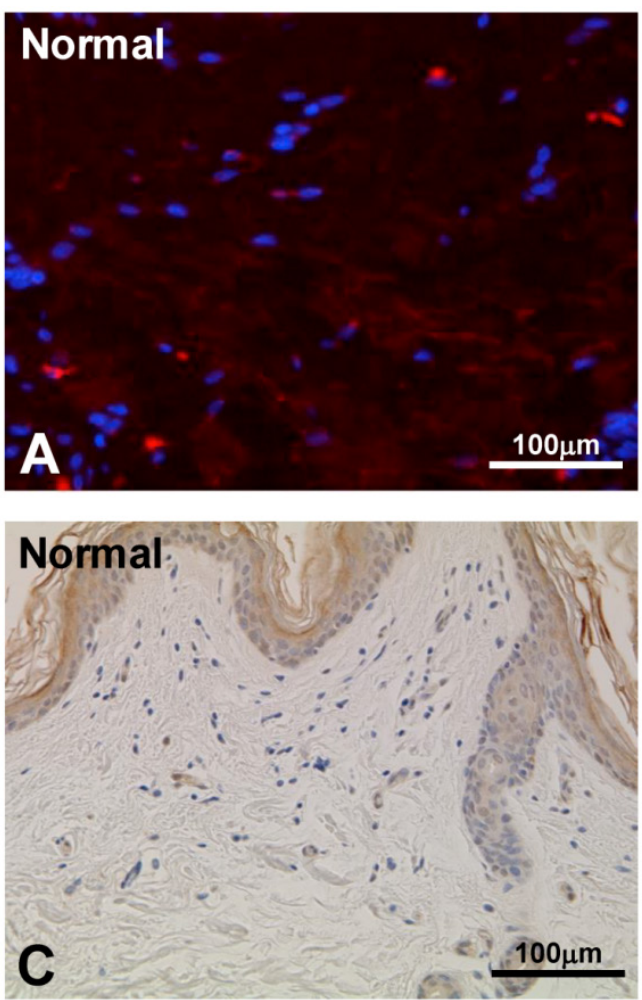
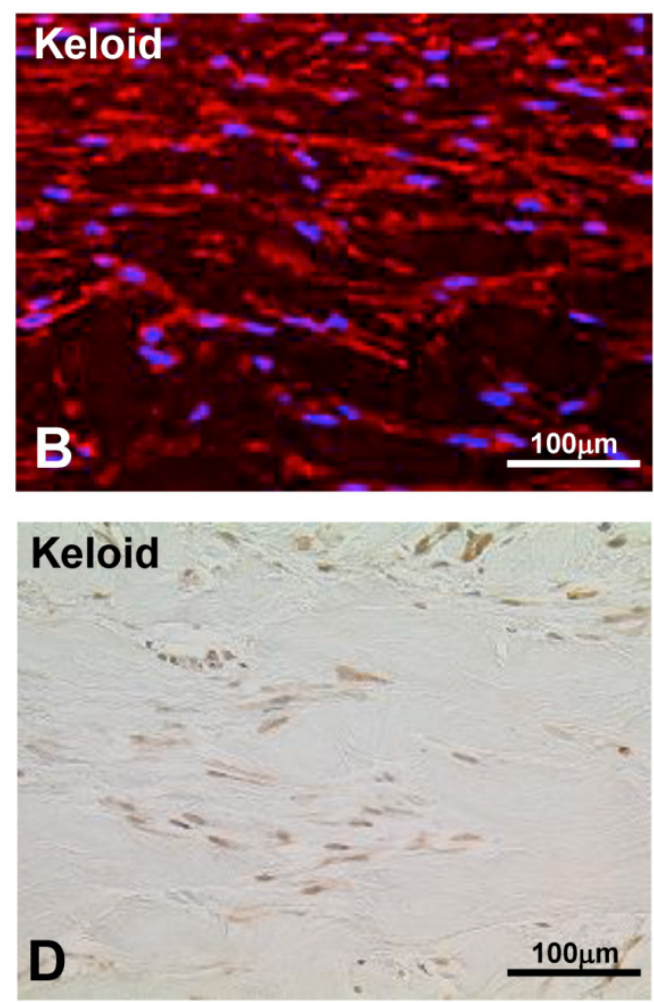

Fig 2. Immunofluorescence staining for $\mathrm{Wnt5a}$ in representative normal skin $(A)$ and keloid tissue (B) demonstrate higher expressions in keloid fibroblasts. Blue nuclear counterstaining was with (DAPI). Both cytoplasmic and occasional nuclear staining was observed. Imunoperoxidase localization of $\mathrm{Wnt5a}$ in a representative normal skin (C) and keloid tissue (D) demonstrates increased expression of Wnt5a in keloid fibroblasts (brown stain). Nuclear counterstaining was with hematoxylin. Note traces or no staining in the normal fibroblasts immediately underneath the epidermis (C). 
Table I. Expression of Wnt5a and $\beta$-catenin in normal skin and keloid tissues.

\begin{tabular}{|c|c|c|c|c|c|c|c|c|c|}
\hline & \multirow{2}{*}{$\begin{array}{l}\text { No. of } \\
\text { Cases }\end{array}$} & \multicolumn{3}{|c|}{ Wnt5a } & \multirow[t]{2}{*}{$\mathrm{P}$} & \multicolumn{3}{|c|}{$\beta$-Catenin } & \multirow[t]{2}{*}{$\mathrm{P}$} \\
\hline & & $\begin{array}{l}\text { Neg/Weak } \\
(0-<25 \%)\end{array}$ & $\begin{array}{l}\text { Moderate } \\
(25-50 \%)\end{array}$ & $\begin{array}{l}\text { Strong } \\
(>50 \%)\end{array}$ & & $\begin{array}{l}\text { Neg/Weak } \\
(0-<25 \%)\end{array}$ & $\begin{array}{l}\text { Moderate } \\
(25-50 \%)\end{array}$ & $\begin{array}{l}\text { Strong } \\
(>50 \%)\end{array}$ & \\
\hline Normal & 10 & $7(70 \%)$ & $3(30 \%)$ & $0(0 \%)$ & $0.013^{*}$ & $7(70 \%)$ & $3(30 \%)$ & $0(0 \%)$ & $0.038^{*}$ \\
\hline Keloid & 18 & $4(22 \%)$ & $3(17 \%)$ & $11(61 \%)$ & & $5(17 \%)$ & $4(28 \%)$ & $9(50 \%)$ & \\
\hline Total & 28 & $11(39 \%)$ & $6(21 \%)$ & $11(39 \%)$ & & $12(43 \%)$ & $7(25 \%)$ & $9(32 \%)$ & \\
\hline
\end{tabular}

* Fisher's exact test: normal vs. keloid.
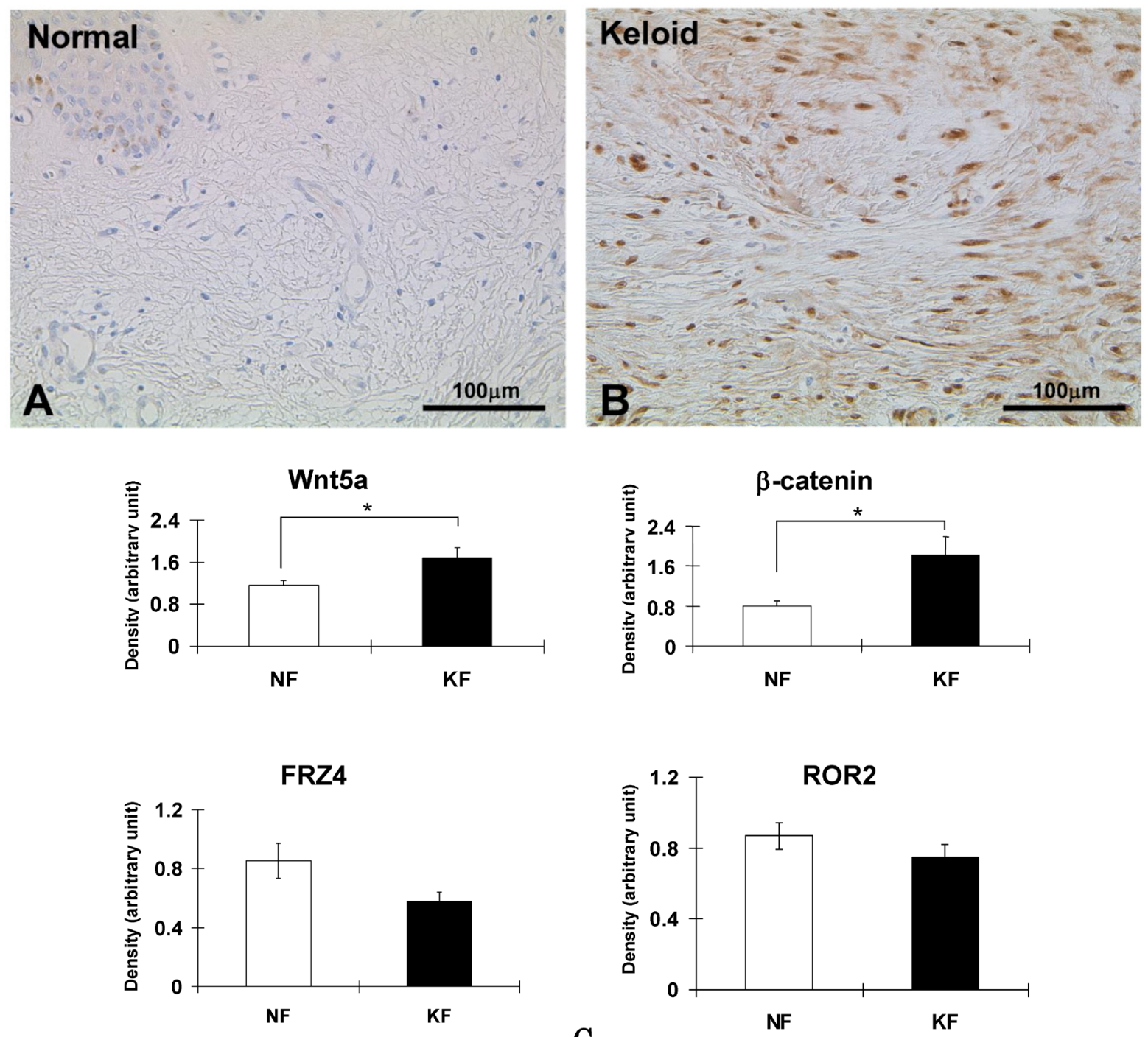

Fig 3. Immunoperoxidase stainings for $\beta$-catenin in a representative normal (A) and keloid (B) tissues show appreciable expression in keloid fibroblasts (B; brown stain). Nuclear counterstaining was with hematoxylin. Note weak or no staining in the normal fibroblasts immediately underneath the epidermis (A). (C) Semi-quantitative Western blot analyses for Wnt5a, $\beta$-catenin FZD4, and ROR2 in the three NF and three KF cases of NF show markedly increased expression of Wnt5a and $\beta$-catenin in $K F(p<0.05)$. 
A

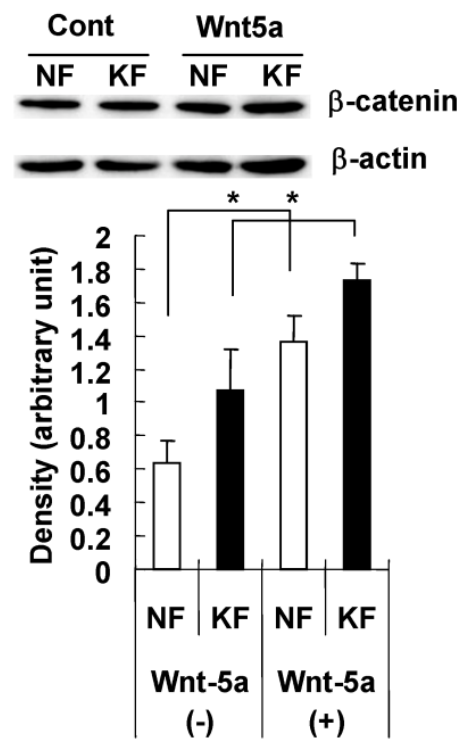

B
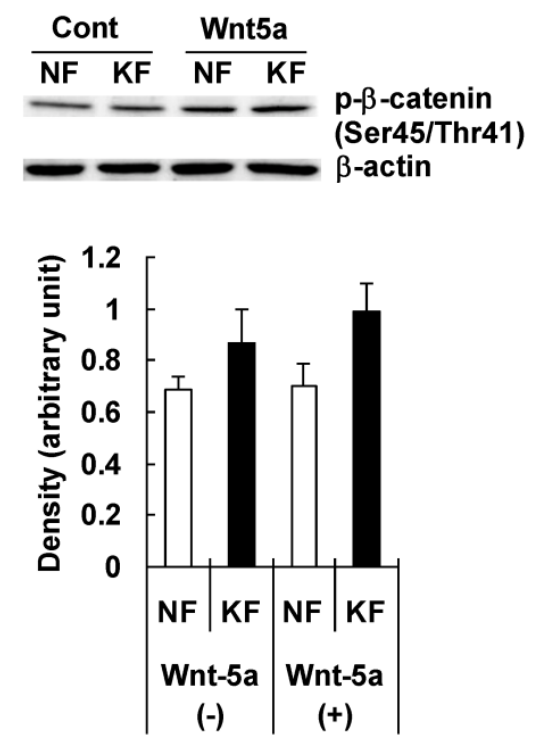

C
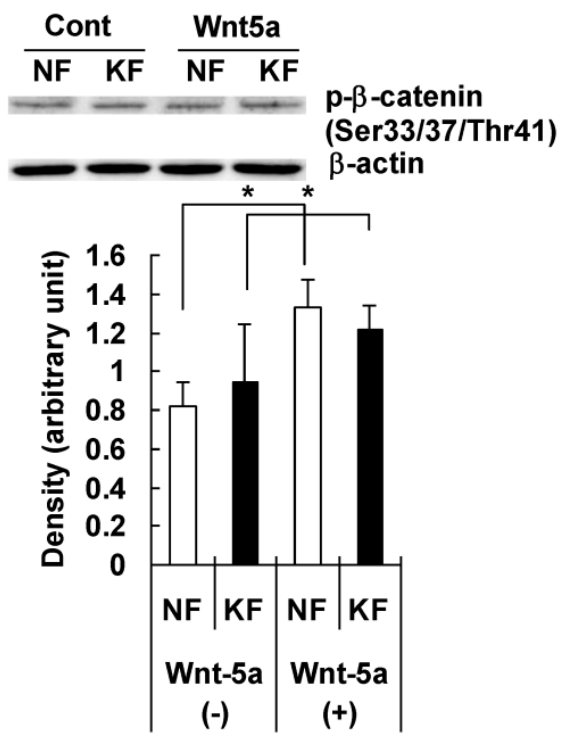

Fig 4. NF and KF cells were treated with $\mathrm{Wnt} 5 \mathrm{a}(3 \mu \mathrm{g} / \mathrm{ml})$ for 72 hours and equal amounts of cell lysates were analyzed for total $\beta$-catenin and phosphorylated $\beta$-catenin at Ser45/Thr4I and at Ser33/37/Thr4I positions and total GSK3- $\beta$ and phosphorylated GSK3- $\beta$ at Ser 9 position using Western blot analyses. Both NF and KF showed a significant increase (relative to $\beta$-actin; $p<0.05$ ) in the total $\beta$-catenin (Fig. $4 \mathrm{~A}$ ), phosphorylated $\beta$-catenin at Ser33/37/Thr4I position (Fig. $2 \mathrm{C}$ ). No significant change was found in phosphorylated $\beta$-catenin at Ser45/Thr4I (Fig. 4B). The Western blot bands are representative for one NF and one KF case. Data represent the mean \pm SD of triplicate measurements from the three NF and three KF cases.

A
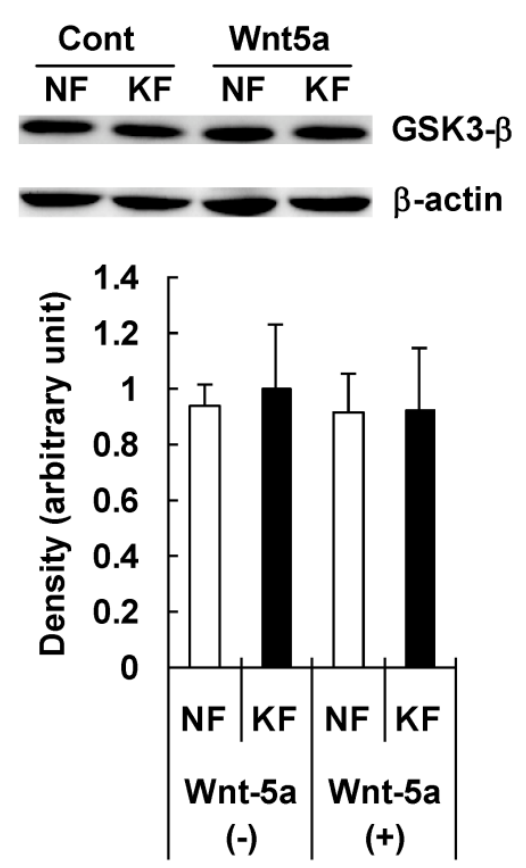

B
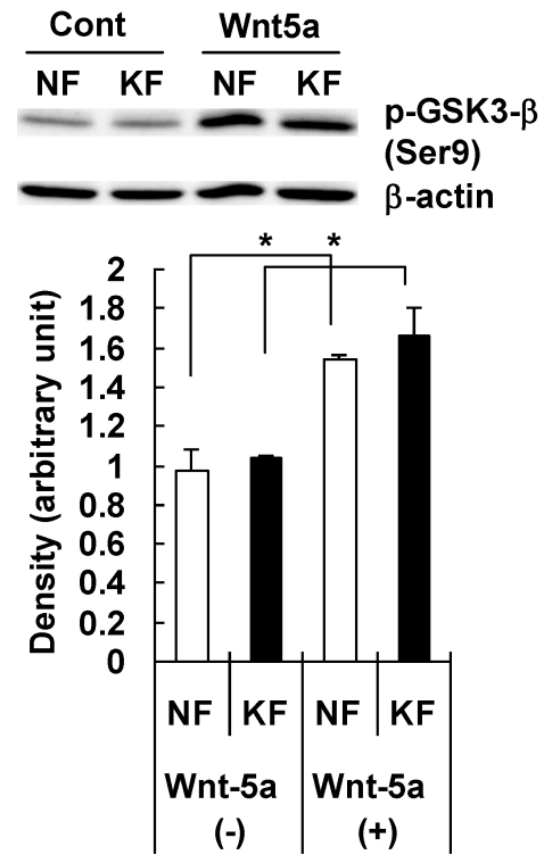

Fig 5. NF and KF cells were treated with Wnt5a $(3 \mu \mathrm{g} / \mathrm{ml})$ for 72 hours and equal amounts of cell lysates were analyzed for total GSK3- $\beta$ and phosphorylated GSK3- $\beta$ at Ser 9 position using Western blot analyses. Both NF and KF showed a significant increase (relative to $\beta$-actin; $p<0.05$ ) in the phosphorylated/inactivated GSK3- $\beta$ level (Fig. 5B). No significant change was found in total GSK3- $\beta$ (Fig. 5A). The Western blot band bands are representative for one NF and one KF case. Data represent the mean \pm SD of triplicate measurements from the three NF and three KF cases. 


\section{Discussion}

Keloid develops as a result of an abnormal wound healing that does not regress spontaneously and frequently recur after surgical excision. The prominent histological characteristics of keloid are fibroblastic proliferation and excessive collagen deposition associated with inflammatory reactions. Many factors such as skin tension, wound infection, racial difference, and genetic predisposition have been implicated in the development of keloid however the exact etiology is still unclear (1). Numerous studies have shown that the Wnt signaling pathway plays a key role in various cellular functions including proliferation, differentiation, survival, apoptosis and migration. Because of the similar features that appear also applicable to the development of keloid, we chose to clarify the role of Wnt signaling pathway in keloid pathogenesis.

In our study, of the Wnt family members, Wnt5a showed an increase at mRNA and protein levels in KF as compared to NF. This finding suggested the involvement of a Wnt5a mediated signaling pathway in keloid pathogenesis. A previous study showed that Wnt5a was significantly increased in fibroblasts obtained from usual interstitial pneumonia (UIP) lung fibroblasts (33). UIP and keloid fibroblasts share common features in terms of fibroblastic cell proliferation and collagen synthesis though their sources of origin are different.

Inflammation plays a major role in the development of Keloid. In view of this tendency, Wnt5a is expressed in human inflammatory diseases (35) including psoriasis, atherosclerosis plaques and rheumatoid arthritis, but not in normal tissue. Psoriatic lesions of the skin were shown to express increased Wnt5a in both the epidermis and dermis compared with control and uninvolved skin by immunohistochemical analysis (36). Human atherosclerotic plaques expressed Wnt5a with predominant staining in the areas of macrophage accumulation within the intima (37). In rheumatoid arthritis, synovial tissues expressed higher level of Wnt5a and cultured synovial fibroblasts expressed higher levels of inflammatory cytokines such as IL-6, IL-8, and IL-15 (38). These data support the biological impact of Wnt5a in other inflammatory lesions and keloid as well. To determine which Wnt signaling pathway operates in keloid fibroblasts, we assessed the expression of $\beta$-catenin and GSK3- $\beta$ which are two major players in the Wnt signaling pathways. In our study, the expression of $\beta$-catenin was increased in keloid fibroblasts and tissues compared to the normal fibroblasts. Previous studies showed that $\beta$-catenin protein expression is increased in keloid tissues and also Wnt signaling and its effecter $\beta$-catenin play an important role in wound healing $(29,39)$. Wounding of $\beta$-catenin overexpressing mice resulted in hyperplastic scar formation (40). Cell cultures derived from patients with normal wound and hyperplastic scar showed transcriptional activation of $\beta$-catenin-TCF/Lef (41). Hyperplastic wound formation was also shown to be dependent on $\beta$-catenin expression and $\beta$-catenin was suggested to regulate wound size and mediates the effect of TGF- $\beta$ in cutaneous healing (42). Furthermore, $\beta$-catenin associated with its cytoplasmic accumulation is frequently observed in fibroproliferative diseases with characteristics of dysregulated wound healing. These diseases include hypertrophic scar formation, aggressive fibromatosis, Lederhose disease, and Dupuytren's contracture (DC) (43). Taking together, our finding of a high expression of $\beta$-catenin in keloid fibroblasts adds support to its critical role in fibroblastic cell proliferation.

Wnt signaling requires inhibition of GSK3- $\beta$ kinase activity to allow accumulation of $\beta$-catenin and resultant gene expression. In our study, in both NFs and KFs, treatment with Wnt5a induced a significant increase in the total $\beta$-catenin level but not the phosphorylated $\beta$-catenin levels at serine 45 and threonine 41 positions, where it is phosphorylated by casein kinase 1a to undergo degradation. Also, there was an increase in phosphorylation of $\beta$-catenin levels at the serine 33, 37, or threonine 41 positions, as would be expected if Wnt5a was active through the canonical Wnt/ $\beta$-catenin pathway. Furthermore, there was no change in the total GSK3- $\beta$ level. Instead, Wnt5a induced a significant increase in the phosphorylated GSK3- $\beta$ at Ser 9 position where it is phosphorylated to become inactive. Unlike most protein kinases involved in signaling, GSK3- $\beta$ is active in unstimulated, resting cells and its activity is diminished during cellular responses. It has preference for primed substrates, that is, substrates previously phosphorylated by another kinase (44). Growth factors and insulin signaling inhibit the ability of GSK3 to act on pre-phosphorylated or primed substrates by phosphorylating Ser9 of GSK3, which then blocks the interaction of GSK3 with the phosphate group on primed substrates (45). In our study, the difference in response to Wnt5a recombinant protein in terms of phosphorylated/inactivated GSK3- $\beta$ level was not significant between NF and KF, although, KF showed a slightly higher trend. As keloid is a constantly slow growing fibroproliferative lesion, even a slight increase in phosphorylated/inactivated GSK3- $\beta$ level could facilitate such functional behavior. Taken to- 
gether, our finding of increased phosphorylation of GSK3- $\beta$ after Wnt5a treatment in both NFs and KFs indicates its increased inactivation or inhibition as seen in the canonical Wnt/ $\beta$-catenin signaling pathway.

FZD4 and ROR2 receptors are two important elements involved in other Wnt signaling pathways (46-48). FZD4 is considered to play a major role in non-canonical or $\beta$-catenin independent Wnt signaling where it was shown to activate protein kinase $C$ (46) and ROR2 is involved in a Wnt signaling pathway mediated through Cdc42 and JNK to regulate downstream target genes transcription $(47,48)$. To investigate whether these pathways might be involved in $\mathrm{KF}$, we assessed the expression of FZD4 and ROR2 proteins. We found no detectable level of both proteins in NF or KF, suggesting no potential role for these receptors. Previous studies showed that expression of ROR2 is required for Wnt5a-mediated inhibition of the canonical Wnt/ $\beta$-catenin signaling. These data exclude the involvement of $\beta$-cateninindependent or non-canonical Wnt/ $\beta$-catenin signaling in KF.

There is increasing evidence to show that the canonical Wnt/ $\beta$-catenin signaling is involved in keloid pathogenesis (28). Sato (29) reported that TGF- $\beta$ induced the upregulation of $\mathrm{Wnt} / \beta$-catenin signaling in hypertrophic scar and keloid fibroblasts. Gene profiling of normal scar fibroblasts and KFs (30) as well as immunohistochemical examination of active areas of keloid tissues (31) revealed the decreased expression of secreted frizzled-related protein 1 (SFRP1), a known Wnt inhibitor. In addition, preliminary microarray profiling of human skin NF and KF performed in our laboratory also revealed significant down-regulation of SFRP1 in KF (unpublished data). In our study, treatment of NF and KF with Wnt5a recombinant protein induced a significant increase in total $\beta$-catenin, phosphorylated $\beta$-catenin at Ser33/37/Thr 41, and phosphorylated/inactivated GSK3- $\beta$. These events might lead to the blockage of $\beta$-catenin phosphorylation and prevent its degradation leading to a Wnt5a/ $\beta$-catenin signaling activation.

Based on our results, a proposed model of Wnt $5 \mathrm{a} / \beta$-catenin signaling in keloid development could be presented as depicted in Figure 6. In KF, binding of the Wnt5a to FZD receptors and LRP5/ 6 activates Dvl which in turn inhibits $\beta$-catenin destruction complex (APC, GSK3- $\beta$, Axin) by increased phosphorylation of GSK3- $\beta$ at Ser 9 position (GSK3- $\beta$ inactivation). This results in the inhibition of $\beta$-catenin phosphorylation and accumulation of $\beta$-catenin in the cytoplasm followed by translocation into the nucleus to regulate the target gene transcription in cooperation with TCF and LEF family transcription factors. As a result, fibroblast cell proliferation and collagen synthesis persist in a slow and repetitive manner leading to the formation of keloid.

In conclusion, our results highlight a potential role for Wnt5a/ $\beta$-catenin canonical pathway in determining the fibroblast phenotype in keloid. Targeting Wnt5a/ $\beta$-catenin signaling may facilitate development of new therapeutic strategies for keloid.

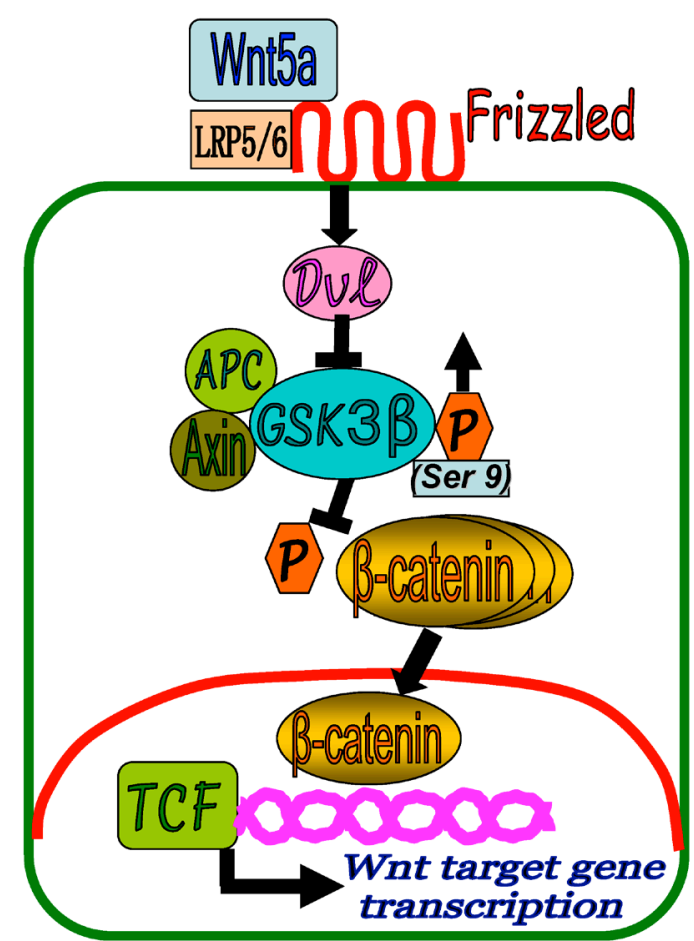

Fig 6. A proposed schematic representation of $\mathrm{Wnt} 5 \mathrm{a} / \beta$-catenin signaling in keloid pathogenesis. In KF, binding of the $\mathrm{Wnt5a}$ to FZD receptors and LRP5/6 activates Dvl which in turn inhibits $\beta$-catenin destruction complex (APC, GSK3- $\beta$, Axin) by increased phosphorylation of GSK3- $\beta$ at Ser 9 position (GSK3- $\beta$ inactivation) leading to the accumulation of $\beta$-catenin in the cytoplasm and translocation into the nucleus to regulate target gene transcription in cooperation with TCF and LEF family transcription factors.

\section{Acknowledgements}

This work was supported in part by Grant-in-Aid (No. 24592722) to Mohammad Ghazizadeh from the Ministry of Education, Culture, Sports, Science and Technology of Japan.

\section{Authors' contributions}

SI and MT prepared tissue and patient data, carried out experiments, interpreted data and co-drafted 
the manuscript. SE performed experiments and generated data. HS performed RT-PCR. MM and $\mathrm{HH}$ provided tissues and patient data for the study. MG conceived and designed the experiments, supervised the study, analyzed data and edited the manuscript.

\section{Conflicts of interest} interest.

The authors declare that there are no conflicts of

\section{References}

1. Al-Attar A, Mess S, Thomassen JM, Kauffman CL, Davison SP. Keloid pathogenesis and treatment. Plast Reconstr Surg. 2006; 117: 286-300

2. Sclafani AP, Gordon L, Chadha M, Romo T 3rd. Prevention of earlobe keloid recurrence with postoperative corticosteroid injections versus radiation therapy: a randomized, prospective study and review of the literature. Dermatol Surg. 1996; 22: 569-574

3. Lo TC, Seckel BR, Salzman FA, Wright KA. Single-dose electron beam irradiation in treatment and prevention of keloids and hypertrophic scars. Radiother Oncol. 1990; 19(3): 267-272

4. Mitsuhashi and Miyashita. Treatment of so-called keloid with excision and postoperative electron irradiation. Nihon Ika Daigaku Zasshi. 1995; 62: 186-195

5. Maarouf M, Schleicher U, Schmachtenberg A, Ammon J. Radiotherapy in the management of keloids. Clinical experience with electron beam irradiation and comparison with X-ray therapy. Strahlenther Onkol. 2002; 178(6): 330-335

6. Tosa M, Ghazizadeh M,_Shimizu H, Hirai T, Hyakusoku H, Kawanami O. Global Gene Expression Analysis of Keloid Fibroblasts in Response to Electron Beam Irradiation Reveals the Involvement of Interleukin-6 Pathway. J Invest Dermatol. 2005; 124: 704 -713

7. Logan, C.Y. \& Nusse, R. The Wnt signaling pathway in development and disease. Ann Rev Cell Dev Biol. 2004; 20: 781-810

8. Nusse, R. Wnt signaling in disease and in development. Cell Res. 2005; 15: $28-32$

9. Angers, Stephane, and Randall T. Moon. Proximal events in Wnt signal transduction. Nat Rev Mol Cell Biol. 2009; 10: 468-477

10. Huelsken, J. \& Birchmeier, W. New aspects of Wnt signaling pathways in higher vertebrates. Curr Opin Genet Dev. 2001; 11: 547-553

11. Nusse, R. Wnts and Hedgehogs: lipid-modified proteins and similarities in signaling mechanisms at the cell surface. Development. 2003; 130: 5297-5305

12. Kühl M, Sheldahl LC, Malbon CC, Moon RT. $\mathrm{Ca}(2+)$ /calmodulin-dependent protein kinase II is stimulated by Wnt and Frizzled homologs and promotes ventral cell fates in Xenopus. J Biol Chem. 2000; 275: 12701-12711

13. Niehrs C. Norrin and frizzled; a new vein for the eye. Dev Cell. 2004; 6:453-454.

14. Winklbauer R, Medina A, Swain RK, Steinbeisser H. Frizzled-7 signalling controls tissue separation during Xenopus gastrulation. Nature. 2001; 413: 856-860

15. Malbon CC. Frizzleds: new members of the superfamily of G-protein-coupled receptors. Front Biosci. 2004; 9: 1048-1058

16. Topol L, Jiang X, Choi H, Carrett-Beal L, Carolan PJ, and Yang Y. Wnt5a inhibits the canonical Wnt pathway by promoting GSK-3 independent B-catenin degradation. J Cell Biol. 2003; 162: 899-908

17. Foord, S.M., Bonner, T.I., Neubig, R.R. International union of pharmacology. XLVI. G protein-coupled receptor list. Pharmacol Rev. 2005; 57: 279-288

18. Nusse R. Wnts and Hedgehogs: lipid-modified proteins and similarities in signaling mechanisms at the cell surface. Development. 2003; 130: 5297-5305

19. Gordon MD, Nusse R. Wnt signaling: multiple pathways, multiple receptors, and multiple transcription factors. J Biol Chem. 2006; 281: 22429-22433

20. Fukuda T, Chen L, Endo T, Tang L, Lu D, Castro JE, Widhopf GF 2nd, Rassenti LZ, Cantwell MJ, Prussak CE, Carson DA, Kipps TJ. Antisera induced by infusions of autologous Ad-CD154-leukemia B cells identify ROR1 as an oncofetal antigen and receptor for Wnt5a. Proc Natl Acad Sci U S A. 2008; 105: 3047-3052

21. Green JL, Kuntz SG, Sternberg PW. Ror receptor tyrosine kinases: orphans no more. Trends Cell Biol. 2008; 18:536-544
22. Kikuchi A, Yamamoto H, Sato A. Selective activation mechanisms of Wnt signaling pathways. Trends Cell Biol. 2009; 19: 119-129

23. Malbon, C.C. \& Wang, H.Y. Dishevelled a mobile scaffold catalyzing development. Curr Top Dev Biol. 2006; 72: 153-166

24. Peifer and Polakis. Wnt signaling in oncogenesis and embryogenesis--a look outside the nucleus. Science. 2000; 287: 1606-1609

25. Chan SK, Struhl G.. Evidence that Armadillo transduces wingless by mediating nuclear export or cytosolic activation of Pangolin. Cell. 2002; 111: 265-280

26. Bienz $M$, Clevers $H$. Armadillo/beta-catenin signals in the nucleus--proof beyond a reasonable doubt? Nat Cell Biol. 2003; 5: 179-182

27. Tolwinski NS, Wehrli M, Rives A, Erdeniz N, DiNardo S, Wieschaus E. $\mathrm{Wg} / \mathrm{Wnt}$ signal can be transmitted through arrow/LRP5,6 and Axin independently of Zw3/Gsk3beta activity. Dev Cell. 2003; 4: 407-418

28. Chua, AW, Ma D, Gan SU, Fu Z, Han HC, Song C et al. The role of R-Spondin2 in keratinocyte proliferation and epidermal thickening in keloid scarring. J Invest Dermatol. 2011; 131: 644-654

29. Sato M. Upregulation of the Wnt/beta-catenin pathway induced by transforming growth factor-beta in hypertrophic scars and keloids. Acta Derm Venereol, 2006; 86: 300-307

30. Smith JC, Boone BE, Opalenik SR, Williams SM, Russell SB. Gene profiling of keloid fibroblasts shows altered expression in multiple fibrosis-associated pathways. J Invest Dermatol. 2008; 128: 1298-1310

31. Russell SB, Russell JD, Trupin KM, Gayden AE, Opalenik SR, Nanney LB et al. Epigenetically altered wound healing in keloid fibroblasts. J Invest Dermatol, 2010; 131: 2489-2496

32. Ghazizadeh M, Tosa M, Shimizu H, Hyakusoku H, Kawanami O. Functional implications of the IL-6 signaling pathway in keloid pathogenesis. J Invest Dermatol. 2007; 127: 98-105

33. Vuga LJ, Ben-Yehudah A, Kovkarova-Naumovski E, Oriss T, Gibson KF, Feghali-Bostwick C, Kaminski N. WNT5A Is a Regulator of fibroblast proliferation and resistance to apoptosis. Am J Resp Cell Mol Biol. 2009; 41: 583-589

34. Ghazizadeh M, Ogawa H, Sasaki Y, Araki T, Aihara K. Mucin carbohydrate antigens $(\mathrm{T}, \mathrm{Tn}$, and sialyl-Tn) in human ovarian carcinomas: relationship with histopathology and prognosis. Human Pathol. 1997; 28: 960-966.

35. George SJ. Wnt pathway: a new role in regulation of inflammation. Arterioscler Thromb Vasc Biol. 2008; 28: 400-402.

36. Gudjonsson JE, Johnston A, Stoll SW, Riblett MB, Xing X, Kochkodan JJ, Ding J, Nair RP, Aphale A, Voorhees JJ, Elder JT. Evidence for altered Wnt signaling in psoriatic skin. J Invest Dermatol. 2010; 130: 1849-1859

37. Christman MA 2nd, Goetz DJ, Dickerson E, McCall KD, Lewis CJ, Benencia F, Silver MJ, Kohn LD, Malgor R. Wnt5a is expressed in murine and human atherosclerotic lesions. Am J Physiol Heart Circ Physiol. 2008; 294: H2864-2870

38. Sen M, Lauterbach K, El-Gabalawy H, Firestein GS, Corr M, Carson DA. Expression and function of wingless and frizzled homologs in rheumatoid arthritis. Proc Natl Acad Sci U S A. 2000; 97: 2791-2796

39. Chua AW, Gan SU, Ting Y, Fu Z, Lim CK, Song C, Sabapathy K, Phan TT. Keloid fibroblasts are more sensitive to Wnt3a treatment in terms of elevated cellular growth and fibronectin expression. J Dermatol Sci. 2011; 64: 199-209

40. Cheon SS, Cheah AY, Turley S, Nadesan P, Poon R, Clevers H, Alman BA. Beta-Catenin stabilization dysregulates mesenchymal cell proliferation, motility, and invasiveness and causes aggressive fibromatosis and hyperplastic cutaneous wounds. Proc Natl Acad Sci USA. 2002; 99: 6973-6978

41. Cheon SS, Nadesan P, Poon R, Alman BA. Growth factors regulate beta-catenin-mediated TCF-dependent transcriptional activation in fibroblasts during the proliferative phase of wound healing. Exp Cell Res. 2004; 293: 267-274

42. Cheon SS, Wei Q, Gurung A, Youn A, Bright T, Poon R, Whetstone H, Guha A, and Alman BA. Beta-catenin regulates wound size and mediates the effect of TGF-beta in cutaneous healing. The FASEB Journal. 2006; 20: 692-701

43. Bowley E, O'Gorman DB, Gan BS. Beta-catenin signaling in fibroproliferative disease. J Surg Res. 2007; 138: 141-150

44. Doble BW,Woodgett JR. GSK-3: tricks of the trade for a multi-tasking kinase. J Cell Sci. 2003; 116: 1175-1186

45. Ferkey DM,Kimelman D. Glycogen synthase kinase-3 beta mutagenesis identifies a common binding domain for GBP and Axin. J Biol Chem. 2002; 277: 16147-16152

46. Robitaille J, MacDonald ML, Kaykas A, et al: Mutant frizzled-4 disrupts retinal angiogenesis in familial exudative vitreoretinopathy. Nat Genet 32: $326-330,2002$ 
47. Yuzugullu H, Benhaj K, Ozturk N, Senturk S, Celik E, Toylu A, Tasdemir N, Yilmaz M, Erdal E, Akcali KC, Atabey N and Ozturk M. Canonical Wnt signaling is antagonized by noncanonical Wnt5a in hepatocellular carcinoma cells. Mol Cancer 2009, 8:90

48. Yuan Y, Niu CC, Deng G, Li ZQ, Pan J, Zhao C, Yang ZL, Si WK. The Wnt5a/Ror2 noncanonical signaling pathway inhibits canonical Wnt signaling in K562 cells. Int J Mol Med. 2011; 27: 63-69. 